\title{
Power functions and essentials of fractional calculus on isolated time scales
}

Tomáš Kisela*

\section{"Correspondence:}

kisela@fme.vutbr.cz

Institute of Mathematics, Brno

University of Technology,

Technická 2, Brno, 616 69, Czech

Republic

\section{Springer}

\begin{abstract}
This paper is concerned about a recently suggested axiomatic definition of power functions on a general time scale and its consequences to fractional calculus. Besides a discussion of the existence and uniqueness of such functions, we derive an efficient formula for the computation of power functions of rational orders on an arbitrary isolated time scale. It can be utilized in the introduction and evaluation of fractional sums and differences. We also deal with the Laplace transform of such fractional operators, which, apart from solving of fractional difference equations, enables a more detailed comparison of our results with those in the relevant literature. Some illustrating examples (including special fractional initial value problems) are presented as well.
\end{abstract}

MSC: $26 \mathrm{E} 70 ; 39 \mathrm{~A} 12 ; 26 \mathrm{~A} 33 ; 44 \mathrm{~A} 10$

Keywords: fractional calculus; power functions; time scales; convolution; Laplace transform

\section{Introduction}

In the last decades, continuous fractional calculus, i.e., a calculus dealing with integrals and derivatives of noninteger orders, evolved in a respected mathematical discipline with a number of physical and technical applications (for more information we refer to monographs, e.g., [1, 2]). Its key notion can be represented by the formula for the fractional integral of a real function $f$

$$
{ }_{a} \mathbf{D}^{-\gamma} f(t)=\int_{a}^{t} \frac{(t-\tau)^{\gamma-1}}{\Gamma(\gamma)} f(\tau) \mathrm{d} \tau, \quad t>a, \gamma>0,
$$

where $\gamma$ is an order of integration. Note that the term 'fractional' is used due to a tradition, because (1) allows to utilize not only rational, but also real and, in principle, even complex values of $\gamma$.

Contrary to the continuous fractional calculus, which has been studied for more than 300 years, its discrete counterpart is a rather new topic. It started by the pioneering works $[3,4]$, where fractional operators were introduced for the $q$-calculus and the difference calculus, respectively. Utilizing a current notation, corresponding fractional sums of an appropriate function $f$ can be written as

$$
{ }_{a} \nabla_{q}^{-\gamma} f\left(q^{n} a\right)=\sum_{k=1}^{n}\left(1-q^{-1}\right) q^{k} a \frac{\left(q^{n} a-q^{k-1} a\right)_{q}^{(\gamma-1)}}{\Gamma_{q^{-1}}(\gamma)} f\left(q^{k} a\right), \quad n \in \mathbb{Z}, \gamma>0
$$

C 2013 Kisela; licensee Springer. This is an Open Access article distributed under the terms of the Creative Commons Attribution License (http://creativecommons.org/licenses/by/2.0), which permits unrestricted use, distribution, and reproduction in any medium, provided the original work is properly cited. 
$(q>0, q \neq 1, a>0)$ and

$$
{ }_{a} \nabla^{-\gamma} f(n)=\sum_{k=1}^{n} \frac{(n-k+1)^{(\gamma-1)}}{\Gamma(\gamma)} f(k), \quad n \in \mathbb{Z}, \gamma>0 .
$$

For more information on definitions of discrete power functions $(\cdot)_{q}^{(\gamma)}$ and $(\cdot)^{(\gamma)}$ we refer, e.g., to $[5,6]$.

In the last decade, many authors proceeded with these ideas and studied properties of corresponding fractional difference equations as well as further generalizations of this theory, e.g., to $h$-calculus and $(q, h)$-calculus (see, e.g., $[5,7-11]$ and [12-14]). In these papers, the authors often preferred the notation based on the time scales theory, which easily exposes similarities among the results derived in $q$-calculus, $h$-calculus, $(q, h)$-calculus and the continuous case. However, this notation was employed only formally, since there was no general time scale definition of the power function occurring in (1)-(3), therefore, the achieved results could not be generally applied to other time scales.

On this account, some ideas regarding fundamental properties which should be met by power functions on time scales were outlined in [15]. In [16], the authors introduced fractional calculus (and by extension also power functions) via the Laplace transform. However, this approach is limited to settings with the well-defined Laplace transform (see [17]) and suffers by some technical difficulties, connected to the inverse Laplace transform. Recently, in [6] and [18], the authors independently suggested an axiomatic definition of power functions on an arbitrary time scale.

It this paper, we are going to continue in this research and discuss this definition and some of its consequences. In Section 2, we recall basics of the time scales theory and present some advanced results concerning a convolution and Laplace transform on time scales. Section 3 is devoted to fractional calculus on time scales. In particular, we state the definition of power functions and fractional operators, comment on their relations to approaches in $[6,15]$ and [18], and point out several interesting properties. In Section 4, we show that the suggested definition is well-posed on every isolated time scale and provides an effective formula for computing values of power functions of rational orders. We also discuss a singular behaviour of negative-order power functions implied by proposed axioms. Section 5 provides a link between our axiomatic definition and the Laplace transform approach developed in [16]. We also present a few examples involving special fractional difference equations, considered on various time scales. The paper is concluded by Section 6, summarizing the obtained results and discussing some induced open problems.

\section{Time scales preliminaries}

A time scale $\mathbb{T}$ is an arbitrary nonempty closed subset of real numbers, e.g., the reals $\mathbb{R}$, arithmetic sequence $h \mathbb{Z}=\{n h ; n \in \mathbb{Z}\}(h>0)$, geometric sequence $\overline{q^{\mathbb{Z}}}=\operatorname{cl}\left\{q^{n} ; n \in \mathbb{Z}\right\}(q>$ $0, q \neq 1$ ) or their combinations denoted as $\mathbb{T}_{(q, h)}$ (see [5]). If inf $\mathbb{T}=a>-\infty$, we denote $\mathbb{T}_{\kappa}=\mathbb{T} \backslash a$, otherwise $\mathbb{T}_{\kappa}=\mathbb{T}$. For any $t \in \mathbb{T}$, we define the forward and backward jump operator $\sigma(t)=\inf \{\tau \in \mathbb{T} ; \tau>t\}$ and $\rho(t)=\sup \{\tau \in \mathbb{T} ; \tau<t\}$, respectively. If $\rho(t)<t<\sigma(t)$ for all $t \in \mathbb{T}$ such that inf $\mathbb{T}<t<\sup \mathbb{T}$, we speak of an isolated time scale. We also recall the forward and backward graininess function $\mu(t)=\sigma(t)-t$ and $\nu(t)=t-\rho(t)$, respectively. For detailed information on the time scales theory, we refer to $[19,20]$. In the sequel, we 
utilize a notation $(a, b)_{M}=(a, b) \cap M$, where $M$ is an arbitrary subset of reals (analogously for closed intervals).

The symbol $\nabla f(t)$ is the nabla derivative of $f: \mathbb{T} \rightarrow \mathbb{R}$ at $t \in \mathbb{T}_{\kappa}$. For our purposes, it is sufficient to recall its form on isolated time scales when

$$
\nabla f(t)=\frac{f(t)-f(\rho(t))}{v(t)}
$$

and $\nabla^{m} f(t)=\nabla\left(\nabla^{m-1} f(t)\right)(m \in \mathbb{Z}, m>1)$. Similarly, we omit a general definition of nabla integral and specify only its isolated time scale version

$$
\int_{a}^{b} f(\tau) \nabla \tau=\sum_{\tau \in(a, b]_{\mathbb{T}}} v(\tau) f(\tau), \quad \int_{a}^{a} f(\tau) \nabla \tau=0
$$

for $f$ being bounded on $[a, b]_{\mathbb{T}}$. Of course, it is possible to consider also the delta derivatives and integrals. However, the nabla version seems to be more suitable for fractional calculus as outlined, e.g., in [5, 14].

Time scale derivatives and integrals are related by several useful rules (see, e.g., [20]), e.g.,

$$
\begin{aligned}
& \int_{s}^{t} \nabla(f(\tau) g(\tau)) \nabla \tau=\int_{s}^{t} \nabla f(\tau) g(\tau) \nabla \tau+\int_{s}^{t} f(\rho(\tau)) \nabla g(\tau) \nabla \tau, \\
& \int_{s}^{t} \nabla f(\tau) \nabla \tau=f(t)-f(s), \\
& \nabla_{t} \int_{s}^{t} y(t, \tau) \nabla \tau=\int_{s}^{\rho(t)} \nabla_{t} y(t, \tau) \nabla \tau+y(t, t), \\
& \nabla_{s} \int_{s}^{t} y(\tau, s) \nabla \tau=\int_{s}^{t} \nabla_{s} y(\tau, s) \nabla \tau-y(s, \rho(s)),
\end{aligned}
$$

where $f, g$ and $y$ are real functions such that the integrals exist and $\nabla_{t}$ represents the derivative with respect to $t$ (an omitted index implies the derivative with respect to the first variable).

To utilize the time scales theory as a unification of continuous and discrete fractional calculus, we have to introduce reasonable extensions of standard definitions of some related notions (see, e.g., [20]). In this connection, we recall the family of monomials $\hat{h}_{m}$ defined for integers $m$ via the recursion

$$
\begin{aligned}
& \hat{h}_{0}(t, s)=1, \\
& \hat{h}_{m}(t, s)=\int_{s}^{t} \hat{h}_{m-1}(\tau, s) \nabla \tau, \quad m \in \mathbb{Z}^{+} .
\end{aligned}
$$

Now, we present some advanced results, related to the Laplace transform and convolution on time scales. We start with the definition of nabla Laplace transform introduced in [17]. 
Definition 2.1 Let $a \in \mathbb{T}_{\mathcal{L}}$, where $\mathbb{T}_{\mathcal{L}}$ is a time scale such that $\sup \mathbb{T}_{\mathcal{L}}=\infty$ and $\sup \{v(t)$; $t \in$ $\left.\mathbb{T}_{\mathcal{L}}\right\}<\infty$. The Laplace transform of $f:(a, \infty)_{\mathbb{T}_{\mathcal{L}}} \rightarrow \mathbb{R}$ is defined by

$$
\mathcal{L}_{a}\{f\}(z)=\int_{a}^{\infty} f(\tau) \hat{e}_{z}(a, \rho(\tau)) \nabla \tau \quad \text { for } z \in \mathcal{D}_{\mathcal{L}}(f)
$$

where $\mathcal{D}_{\mathcal{L}}(f)$ consists of all $z \in \mathbb{C}$, for which the improper integral exists, and for which $1-z v(t) \neq 0$ for all $t \in(a, \infty)_{\mathbb{T}_{\mathcal{L}}}$. The symbol $\hat{e}_{z}$ denotes the nabla time scale exponential function (see [20]).

Remark 2.2 Clearly, a necessary condition for existence of $\mathcal{L}_{a}\{f\}(z)$ is $\lim _{\tau \rightarrow \infty} f(\tau)$. $e_{z}(a, \rho(\tau))=0$.

By a modification of the proofs presented in $[17,21]$, we can prove analogues of many important properties of the Laplace transform known from continuous analysis. For our purposes, we recall

$$
\begin{aligned}
& \mathcal{L}_{a}\left\{\hat{h}_{m}(\cdot, a)\right\}(z)=z^{-m-1}, \quad m \in \mathbb{Z}_{0}^{+}, \\
& \mathcal{L}_{a}\left\{\nabla^{m} f\right\}(z)=z^{m} \mathcal{L}_{a}\{f\}(z)-\left.\sum_{j=0}^{m-1} z^{j} \nabla^{m-j-1} f(t)\right|_{t=a}, \quad m \in \mathbb{Z}^{+} .
\end{aligned}
$$

Regarding one of key results connected to the Laplace transform, the convolution theorem, we first have to introduce a convolution on time scales. This issue was solved in [22] for the case of the delta calculus. We adapt this approach for the nabla calculus.

Definition 2.3 Let $a \in \mathbb{T}$. The shift of $f:(a, \infty)_{\mathbb{T}} \rightarrow \mathbb{R}$ is defined as the solution of the shifting problem

$$
\begin{aligned}
& \nabla_{t} u(t, \rho(s))=-\nabla_{s} u(t, s), \quad s, t \in \mathbb{T}, t>s>a, \\
& u(t, a)=f(t), \quad t \in \mathbb{T}, t>a .
\end{aligned}
$$

Remark 2.4 (i) If the graininess is constant, the shifting problem has a unique solution $u(t, s)=f(t-s)$.

(ii) For all $\xi \in \mathbb{T}$, the shift of $\hat{h}_{m}(t, \xi), \hat{e}_{\lambda}(t, \xi)\left(m \in \mathbb{Z}_{0}^{+}, \lambda \in \mathbb{R}\right)$ is $\hat{h}_{m}(t, s), \hat{e}_{\lambda}(t, s)$, respectively (see [22]).

Definition 2.5 Let $s, t \in \mathbb{T}$ be such that $t \geq s$. The convolution of $f, g: \mathbb{T} \rightarrow \mathbb{R}$ is defined by

$$
(f * g)(t, s)=\int_{s}^{t} \hat{f}(t, \rho(\tau)) g(\tau) \nabla \tau, \quad \text { where } \hat{f} \text { is the shift of } f
$$

Theorem 2.6 Let $f, g$ be functions such that $\mathcal{L}_{a}\{f\}(z), \mathcal{L}_{a}\{g\}(z)$ exist. Then

$$
\mathcal{L}_{a}\{(f * g)(\cdot, a)\}(z)=\mathcal{L}_{a}\{f\}(z) \cdot \mathcal{L}_{a}\{g\}(z)
$$


Proof Analogous assertion was proved in [22] for the delta calculus. We utilize a similar technique with several adjustments better suiting our purposes.

Utilizing the formulas $\int_{a}^{T} \int_{a}^{t} f(t, \tau) \nabla \tau \nabla t=\int_{a}^{T} \int_{\rho(\tau)}^{T} f(t, \tau) \nabla t \nabla \tau$ and $\hat{e}_{z}(t, s)=\hat{e}_{z}(t, \xi)$. $e_{z}(\xi, s)$ (see [20]), we can write

$$
\begin{aligned}
\mathcal{L}_{a}\{(f * g)(\cdot, a)\}(z) & =\int_{a}^{\infty}(f * g)(t, a) \hat{e}_{z}(a, \rho(t)) \nabla t \\
& =\int_{a}^{\infty} \hat{e}_{z}(a, \rho(t)) \int_{a}^{t} \hat{f}(t, \rho(\tau)) g(\tau) \nabla \tau \nabla t \\
& =\int_{a}^{\infty} g(\tau) \hat{e}_{z}(a, \rho(\tau)) \int_{\rho(\tau)}^{\infty} \hat{e}_{z}(\rho(\tau), \rho(t)) \hat{f}(t, \rho(\tau)) \nabla t \nabla \tau \\
& =\int_{a}^{\infty} g(\tau) \hat{e}_{z}(a, \rho(\tau)) \Psi(\rho(\tau)) \nabla \tau,
\end{aligned}
$$

where $\Psi(s)=\int_{s}^{\infty} \hat{e}_{z}(s, \rho(t)) \hat{f}(t, s) \nabla t$. We show that $\Psi(s)$ is constant, i.e., its derivative is zero. Indeed, employing (8), (5), Definition 2.3, Remark 2.4(ii), (6) and Remark 2.2, yields

$$
\begin{aligned}
\nabla_{s} \Psi(s) & =\nabla_{s} \int_{s}^{\infty} \hat{e}_{z}(s, \rho(t)) \hat{f}(t, s) \nabla t=\int_{s}^{\infty} \nabla_{s}\left(\hat{e}_{z}(s, \rho(t)) \hat{f}(t, s)\right) \nabla t-\hat{f}(s, \rho(s)) \\
& =\int_{s}^{\infty}\left(\nabla_{s} \hat{e}_{z}(s, \rho(t)) \hat{f}(t, \rho(s))+\hat{e}_{z}(s, \rho(t)) \nabla_{s} \hat{f}(t, s)\right) \nabla t-\hat{f}(s, \rho(s)) \\
& =\int_{s}^{\infty}\left(-\nabla_{t} \hat{e}_{z}(s, t) \hat{f}(t, \rho(s))-\hat{e}_{z}(s, \rho(t)) \nabla_{t} \hat{f}(t, \rho(s))\right) \nabla t-\hat{f}(s, \rho(s)) \\
& =-\int_{s}^{\infty} \nabla_{t}\left(\hat{e}_{z}(s, t) \hat{f}(t, \rho(s))\right) \nabla t+\hat{f}(s, \rho(s)) \\
& =-\left[\hat{e}_{z}(s, t) \hat{f}(t, \rho(s))\right]_{s}^{\infty}-\hat{f}(s, \rho(s))=0 .
\end{aligned}
$$

Hence, substituting $\Psi(\rho(\tau))=\Psi(a)$ into (13), we obtain $\mathcal{L}_{a}\{(f * g)(\cdot, a)\}(z)=\mathcal{L}_{a}\{g\}(z)$. $\mathcal{L}_{a}\{f\}(z)$.

\section{Foundations of fractional calculus on time scales}

As indicated by (1)-(3), the issue of fractional calculus in the time scales theory is connected to the problem of defining appropriate power functions. A standard method, based on a generalization of explicit formulas for monomials to noninteger values of $m$, is effective only in special cases $\left(h \mathbb{Z}, \overline{q^{\mathbb{Z}}}, \mathbb{T}_{(q, h)}, \mathbb{R}\right)$. In general, the explicit formulas for monomials are not known, therefore, a different approach is required.

Recently, in [6] and [18], there were independently suggested quite similar axiomatic definitions of time scales power functions. We develop the one presented in [18].

Definition 3.1 Let $s, t \in \mathbb{T}$, and let $\beta, \gamma \in(-1, \infty)$. The time scales power functions $\hat{h}_{\beta}(t, s)$ are defined as a family of nonnegative functions satisfying

(i) $\int_{s}^{t} \hat{h}_{\beta}(t, \rho(\tau)) \hat{h}_{\gamma}(\tau, s) \nabla \tau=\hat{h}_{\beta+\gamma+1}(t, s)$ for $t \geq s$,

(ii) $\hat{h}_{0}(t, s)=1$ for $t \geq s$,

(iii) $\hat{h}_{\beta}(t, t)=0$ for $0<\beta<1$.

Further, the parameter $\beta$ in $\hat{h}_{\beta}(t, s)$ is called the order of the power function $\hat{h}_{\beta}(t, s)$. 
Compared to the definition in [6], the key underlying ideas are basically the same. However, some additional conditions are prescribed in [6]. The main difference lies in the condition $\hat{h}_{\beta}(t, s)=0$, whenever $t \leq s$ for all $\beta>-1$. In our opinion, this assumption creates an inconsistence with the definition of time scales monomials $\left(\hat{h}_{0}(t, s)=1\right.$ for all $\left.s, t \in \mathbb{T}\right)$ and with the well-known power functions on $\mathbb{T}=\mathbb{R}\left(\hat{h}_{\beta}(t, s)=\frac{(t-s)^{\beta}}{\Gamma(\beta+1)}\right.$ is in general undefined for $t<s$ ). Thus, we prefer to leave the values of $\hat{h}_{\beta}(t, s)$ undefined for $t<s$ (they do not occur in fractional calculus anyway). The consequences for $t=s$ will be discussed later. We point out that for $t>s$, our definition coincides with [6, Definition 3.2] and with historically established power functions on $\mathbb{R}, h \mathbb{Z}, \overline{q^{\mathbb{Z}}}, \mathbb{T}_{(q, h)}$ and their subintervals (see [6] and [18]). We note that condition (i) in Definition 3.1 first appeared without a deeper analysis or discussion in [15].

Remark 3.2 The well-posedness of Definition 3.1 was not discussed in [6] nor [18]. In Section 4, we show that it determines uniquely at least the power functions of rational orders on isolated time scales. In the remaining part of the current section, we assume that the power functions on corresponding time scales exist.

Lemma 3.3 Let $m \in \mathbb{Z}^{+}, \beta \in(-1, \infty), s, t \in \mathbb{T}$ be such that $t>s$. Then

$$
\nabla^{m} \hat{h}_{\beta}(t, s)= \begin{cases}\hat{h}_{\beta-m}(t, s), & \beta>m-1, \\ 0, & \beta \in\{0,1, \ldots, m-1\} .\end{cases}
$$

Proof Let $m=1$ and $\beta>0$. Then, utilizing Definition 3.1(i) and (7), we can write $\nabla \hat{h}_{\beta}(t, s)=$ $\nabla \int_{s}^{t} \hat{h}_{\beta-1}(\tau, s) \nabla \tau=\hat{h}_{\beta-1}(t, s)$. The first part of the assertion now follows by the induction principle. The second part is a consequence of $\nabla \hat{h}_{0}(t, s)=0$.

Remark 3.4 Lemma 3.3 does not discuss the case $\beta \in(-1, m-1] \backslash\{0,1, \ldots, m-1\}$ due to an occurrence of power functions of order less than -1 . Since such functions cannot be included in Definition 3.1 (e.g., due to unintegrability in real analysis), the extension of Lemma 3.3 can serve as their definition, e.g.,

$$
\hat{h}_{\beta}(t, s)=\nabla^{-\lceil\beta\rceil} \hat{h}_{\beta-\lceil\beta\rceil}(t, s), \quad \beta \in(-\infty,-1) \backslash \mathbb{Z}, s, t \in \mathbb{T}, t \geq \sigma^{-\lceil\beta\rceil}(s),
$$

where $\lceil\beta\rceil$ is the ceiling function $\lceil\beta\rceil=\min \{m \in \mathbb{Z} ; m \geq \beta\}$. The presented approach does not allow to introduce power functions of negative integer orders.

Considering the well-defined power functions, we can introduce fractional operators in the frame of the time scales theory (see $[6,15]$ and $[12,18]$ ) following the continuous paradigm (see, e.g., [1, 2]).

Definition 3.5 Let $\gamma \geq 0, \alpha>0, \tilde{a}, a, b \in \mathbb{T}$ be such that $\tilde{a} \leq a<b$. Then for a function $f:(\tilde{a}, b]_{\mathbb{T}} \rightarrow \mathbb{R}$ we define

(i) the fractional integral of order $\gamma>0$ with the lower limit $a$ as

$$
{ }_{a} \nabla^{-\gamma} f(t)=\int_{a}^{t} \hat{h}_{\gamma-1}(t, \rho(\tau)) f(\tau) \nabla \tau, \quad t \in[a, b]_{\mathbb{T}} \cap(\tilde{a}, b]_{\mathbb{T}}
$$

and for $\gamma=0$, we put ${ }_{a} \nabla^{0} f(t)=f(t)$, 
(ii) the Riemann-Liouville fractional derivative of order $\alpha$ with the lower limit $a$ as

$$
{ }_{a} \nabla^{\alpha} f(t)=\nabla^{\lceil\alpha\rceil} \nabla^{\alpha-\lceil\alpha\rceil} f(t), \quad t \in[\sigma(a), b]_{\mathbb{T}} \cap(\sigma(\tilde{a}), b]_{\mathbb{T}},
$$

(iii) and the Caputo fractional derivative of order $\alpha$ with the lower limit $a(a>\tilde{a})$ as

$$
{ }_{a}^{C} \nabla^{\alpha} f(t)={ }_{a} \nabla^{\alpha-[\alpha]} \nabla^{\lceil\alpha\rceil} f(t), \quad t \in[\sigma(a), b]_{\mathbb{T}} .
$$

Remark 3.6 (i) For $\gamma=1$, Definition 3.5(i) reduces to a formula for the antiderivative ${ }_{a} \nabla^{-1} f(t)=\int_{a}^{t} f(\tau) \nabla \tau$ known from the time scales theory (see [20]).

(ii) We can see a formal agreement of Definition 3.5(i) with (1)-(3) (for detailed information see [6]).

Remark 3.7 (i) If $a>\tilde{a}$, we obtain a usual definitions of the difference calculus, $q$-calculus and $(q, h)$-calculus. In this case, it holds $\left.{ }_{a} \nabla^{-\gamma} f(t)\right|_{t=a}=0$ on every time scale.

(ii) If $a=\tilde{a}$, the integrated function is undefined at the lower limit of the integral. Such functions play an important role in continuous fractional calculus and, as we demonstrate in the following sections, can appear also on isolated time scales.

In [6] and [18], the authors proved many time scales analogues of important properties of fractional operators known from continuous analysis, e.g., the index $\operatorname{law}_{a} \nabla^{-\gamma} \nabla^{-\beta} f(t)=$ ${ }_{a} \nabla^{-(\gamma+\beta)} f(t)(\gamma, \beta>0)$. One of the key relationships of the fractional calculus is a power rule, which can be written as

Theorem 3.8 Let $\alpha \in \mathbb{R}, \beta \in(-1, \infty)$ and $s, t \in \mathbb{T}$ be such that $t>s$. Then it holds

$$
{ }_{a} \nabla^{\alpha} \hat{h}_{\beta}(t, a)= \begin{cases}\hat{h}_{\beta-\alpha}(t, s), & \beta>\alpha-1, \\ 0, & \beta \in\{\alpha-\lceil\alpha\rceil, \alpha-\lceil\alpha\rceil+1, \ldots, \alpha-1\} .\end{cases}
$$

Proof For $\alpha<0$ it follows by Definitions 3.5(i) and 3.1(i). For $\alpha>0$, we utilize also Lemma 3.3.

By similar arguments, we can derive an analogous formula for Caputo fractional derivative, i.e.,

Theorem 3.9 Let $\alpha>0, \beta \in(-1, \infty)$ and $s, t \in \mathbb{T}$ be such that $t>s$. Then it holds

$$
{ }_{a}^{C} \nabla^{\alpha} \hat{h}_{\beta}(t, a)= \begin{cases}\hat{h}_{\beta-\alpha}(t, s), & \beta>\lceil\alpha\rceil-1, \\ 0, & \beta \in\{0,1, \ldots,\lceil\alpha\rceil-1\} .\end{cases}
$$

\section{Power functions on isolated time scales}

Although the axiomatic definition of power functions has been proposed, its feasibility, i.e., the question of existence and uniqueness, has not been discussed yet. In [6], the author presents examples of such functions on $\mathbb{R}, h \mathbb{Z}, q^{\mathbb{Z}}$ or $\mathbb{T}_{(q, h)}$, but those functions are known historically, they are not originally based on the proposed axiomatic definition.

In this section, we analyze Definition 3.1 on an arbitrary isolated time scale. We constructively prove the existence and uniqueness of rational-order power functions, which 
enables us to present graphs of power functions on some chosen time scales for the first time. Further, we discuss some properties of negative-order power functions, which are, to the author's knowledge, quite new in the frame of isolated time scales.

Lemma 4.1 Let $\beta \in(-1, \infty), s, t \in \mathbb{T}_{\kappa}$ be such that $t>s$. Then $\hat{h}_{\beta}$ solves the shifting problem, i.e.,

$$
\nabla_{t} \hat{h}_{\beta}(t, \rho(s))=-\nabla_{s} \hat{h}_{\beta}(t, s)
$$

Proof For $\beta \in \mathbb{Z}_{0}^{+}$, the result is known (see Remark 2.4(ii)). Thus, for $\beta \in(-1, \infty) \backslash \mathbb{Z}_{0}^{+}$, we can write

$$
\begin{aligned}
0= & \nabla_{t} \hat{h}_{\lceil\beta\rceil+1}(t, \rho(s))+\nabla_{s} \hat{h}_{\lceil\beta\rceil+1}(t, s) \\
= & \nabla_{t} \int_{\rho(s)}^{t} \hat{h}_{\beta}(t, \rho(\tau)) \hat{h}_{\lceil\beta\rceil-\beta}(\tau, \rho(s)) \nabla \tau+\nabla_{s} \int_{s}^{t} \hat{h}_{\beta}(t, \rho(\tau)) \hat{h}_{\lceil\beta\rceil-\beta}(\tau, s) \nabla \tau \\
= & \int_{\rho(s)}^{\rho(t)} \nabla_{t} \hat{h}_{\beta}(t, \rho(\tau)) \hat{h}_{\lceil\beta\rceil-\beta}(\tau, \rho(s)) \nabla \tau+\hat{h}_{\beta}(t, \rho(t)) \hat{h}_{\lceil\beta\rceil-\beta}(t, \rho(s)) \\
& +\int_{s}^{t} \hat{h}_{\beta}(t, \rho(\tau)) \nabla_{s} \hat{h}_{\lceil\beta\rceil-\beta}(\tau, s) \nabla \tau-\hat{h}_{\beta}(t, \rho(s)) \hat{h}_{\lceil\beta\rceil-\beta}(s, \rho(s)),
\end{aligned}
$$

where we employ (7) and (8). Utilizing (6) and (5), we have

$$
\begin{aligned}
\hat{h}_{\beta}(t, \rho(t)) \hat{h}_{\lceil\beta\rceil-\beta}(t, \rho(s))-\hat{h}_{\beta}(t, \rho(s)) \hat{h}_{\lceil\beta\rceil-\beta}(s, \rho(s)) \\
\quad=\int_{s}^{t} \nabla_{\tau}\left(\hat{h}_{\beta}(t, \rho(\tau)) \hat{h}_{\lceil\beta\rceil-\beta}(\tau, \rho(s))\right) \nabla \tau \\
=\int_{s}^{t} \nabla_{\tau} \hat{h}_{\beta}(t, \rho(\tau)) \hat{h}_{\lceil\beta\rceil-\beta}(\rho(\tau), \rho(s)) \nabla \tau+\int_{s}^{t} \hat{h}_{\beta}(t, \rho(\tau)) \nabla_{\tau} \hat{h}_{\lceil\beta\rceil-\beta}(\tau, \rho(s)) \nabla \tau \\
=\int_{\rho(s)}^{\rho(t)} \nabla_{\tau} \hat{h}_{\beta}(t, \tau) \hat{h}_{\lceil\beta\rceil-\beta}(\tau, \rho(s)) \nabla \tau+\int_{s}^{t} \hat{h}_{\beta}(t, \rho(\tau)) \nabla_{\tau} \hat{h}_{\lceil\beta\rceil-\beta}(\tau, \rho(s)) \nabla \tau .
\end{aligned}
$$

Substituting this into (14), we get

$$
\begin{aligned}
& \nabla_{t} \hat{h}_{\lceil\beta\rceil+1}(t, \rho(s))+\nabla_{s} \hat{h}_{\lceil\beta\rceil+1}(t, s) \\
& \quad=\int_{\rho(s)}^{\rho(t)}\left(\nabla_{t} \hat{h}_{\beta}(t, \rho(\tau))+\nabla_{\tau} \hat{h}_{\beta}(t, \tau)\right) \hat{h}_{\lceil\beta\rceil-\beta}(\tau, \rho(s)) \nabla \tau \\
& \quad+\int_{s}^{t} \hat{h}_{\beta}(t, \rho(\tau))\left(\nabla_{s} \hat{h}_{\lceil\beta\rceil-\beta}(\tau, s)+\nabla_{\tau} \hat{h}_{\lceil\beta\rceil-\beta}(\tau, \rho(s))\right) \nabla \tau .
\end{aligned}
$$

Now, using (7) and (8), we evaluate the derivatives of $\hat{h}_{\lceil\beta\rceil-\beta}$, i.e.,

$$
\begin{aligned}
& \nabla_{s} \hat{h}_{\lceil\beta\rceil-\beta}(\tau, s)=\nabla_{s} \int_{s}^{\tau} \hat{h}_{\lceil\beta\rceil-\beta-1}\left(\tau, \rho\left(\tau_{1}\right)\right) \nabla \tau_{1}=-\hat{h}_{\lceil\beta\rceil-\beta-1}(\tau, \rho(s)), \\
& \nabla_{\tau} \hat{h}_{\lceil\beta\rceil-\beta}(\tau, \rho(s))=\nabla_{\tau} \int_{\rho(s)}^{\tau} \hat{h}_{\lceil\beta\rceil-\beta-1}\left(\tau_{1}, \rho(s)\right) \nabla \tau_{1}=\hat{h}_{\lceil\beta\rceil-\beta-1}(\tau, \rho(s)) .
\end{aligned}
$$


Thus, we arrive at

$$
\begin{aligned}
\nabla_{t} \hat{h}_{\lceil\beta\rceil+1}(t, \rho(s))+\nabla_{s} \hat{h}_{\lceil\beta\rceil+1}(t, s) \\
\quad=\int_{\rho(s)}^{\rho(t)}\left(\nabla_{t} \hat{h}_{\beta}(t, \rho(\tau))+\nabla_{\tau} \hat{h}_{\beta}(t, \tau)\right) \hat{h}_{\lceil\beta\rceil-\beta}(\tau, \rho(s)) \nabla \tau=0,
\end{aligned}
$$

which implies the assertion.

Remark 4.2 Lemma 4.1 enables us to write Definition 3.1(i) via the convolution. Indeed, setting $f(t)=\hat{h}_{\beta}(t, s)$ and $g(t)=\hat{h}_{\gamma}(t, s)$, Definition 2.5 yields

$$
\left(\hat{h}_{\beta} * \hat{h}_{\gamma}\right)(t, s)=\hat{h}_{\beta+\gamma+1}(t, s), \quad t \geq s, \beta, \gamma>-1
$$

Theorem 4.3 Let $\mathbb{T}$ be an isolated time scale, and let $r \in(-1, \infty)_{\mathbb{Q}}$. Then Definition 3.1 determines uniquely the power function $\hat{h}_{r}(t, s)$ for all $s, t \in \mathbb{T}$ such that $t>s$.

Proof We prove this assertion constructively. Due to Definition 3.1(ii), the power functions for $r \in \mathbb{Z}_{0}^{+}$reduce to monomials uniquely given by (9), (10). Now, let $r=\frac{u}{w+1}(u \in \mathbb{Z}$, $\left.w \in \mathbb{Z}^{+},-u \leq w\right)$ be the order of the power function and $a, t \in \mathbb{T}$ be such that $t>a$. Subsequent application of Definition 3.1(i) yields

$$
(\underbrace{\hat{h}_{r} * \cdots * \hat{h}_{r}}_{(w+1) \times})\left(t, \rho^{k}(t)\right)=\hat{h}_{u+w}\left(t, \rho^{k}(t)\right) \quad \text { for all } k \text { such that } \rho^{k}(t) \in \mathbb{T} \text {. }
$$

Setting $t=\sigma^{n}(a)$, we expand the left-hand side of the equation (see Definition 2.5 and (4)) and get

$$
\begin{aligned}
& \sum_{i_{1}=n-k+1}^{n} v\left(\sigma^{i_{1}}(a)\right) \hat{h}_{r}\left(\sigma^{n}(a), \sigma^{i_{1}-1}(a)\right) \sum_{i_{2}=n-k+1}^{i_{1}} v\left(\sigma^{i_{2}}(a)\right) \hat{h}_{r}\left(\sigma^{i_{1}}(a), \sigma^{i_{2}-1}(a)\right) \cdots \\
& \quad \sum_{i_{w}=n-k+1}^{i_{w-1}} v\left(\sigma^{i_{w}}(a)\right) \hat{h}_{r}\left(\sigma^{i_{w-1}}(a), \sigma^{i_{w}-1}(a)\right) \hat{h}_{r}\left(\sigma^{i_{w}}(a), \sigma^{n-k}(a)\right) \\
& =\hat{h}_{u+w}\left(\sigma^{n}(a), \sigma^{n-k}(a)\right) .
\end{aligned}
$$

It turns out that the key parameter in solving of this equation is $k$. For $k=1$, we obtain a simple form $v^{w}\left(\sigma^{n}(a)\right)\left(\hat{h}_{r}\left(\sigma^{n}(a), \sigma^{n-1}(a)\right)\right)^{w+1}=\hat{h}_{u+w}\left(\sigma^{n}(a), \sigma^{n-k}(a)\right)$. Utilizing the property of monomials $\hat{h}_{m}(t, \rho(t))=v^{m}(t)\left(m \in \mathbb{Z}_{0}^{+}\right)$, we can write

$$
\hat{h}_{r}(t, \rho(t))=v^{r}(t), \quad t \in \mathbb{T}_{\kappa} .
$$

Note, that only positive values of $\hat{h}_{r}(t, \rho(t))$ are allowed even for $w$ being odd. Indeed, if we consider $\hat{h}_{r}(t, s)=\left(\hat{h}_{\frac{r-1}{2}} * \hat{h}_{\frac{r-1}{2}}\right)(t, s)$ for $s=\rho(t)$, we get $\hat{h}_{r}(t, \rho(t))=v(t)\left(\hat{h}_{\frac{r-1}{2}}(t, \rho(t))\right)^{2}>0$. 
Now let $k>1$. For the sake of brevity we introduce the symbol $\varphi_{n}^{k}=\hat{h}_{r}\left(\sigma^{n}(a), \sigma^{n-k}(a)\right)$. By shifting all indices by $n-k+1$ and rearranging the sums, we get

$$
\begin{aligned}
& \sum_{i_{1}=0}^{k-1} \sum_{i_{2}=0}^{i_{1}} \cdots \sum_{i_{w}=0}^{i_{w-1}}\left(\prod_{\ell=1}^{w} v\left(\sigma^{n-k+1+i_{\ell}}(a)\right)\right)\left(\prod_{\ell=1}^{w-1} \varphi_{n-k+1+i_{\ell}}^{1+i_{\ell}-i_{\ell+1}}\right) \varphi_{n}^{k-i_{1}} \varphi_{n-k+1+i_{w}}^{1+i_{w}} \\
& =\hat{h}_{u+w}\left(\sigma^{n}(a), \sigma^{n-k}(a)\right) .
\end{aligned}
$$

Further, we perform the transformation $i_{1} \rightarrow k-1-i_{1}, i_{2} \rightarrow k-1-i_{1}-i_{2}$ etc. to obtain

$$
\begin{aligned}
& \sum_{i_{1}=0}^{k-1} \sum_{i_{2}=0}^{k-1-i_{1}} \cdots \sum_{i_{w}=0}^{k-1-\sum_{p=1}^{w-1} i_{p}}\left(\prod_{\ell=1}^{w} v\left(\sigma^{n-\sum_{p=1}^{\ell} i_{p}}(a)\right) \varphi_{n-\sum_{p=1}^{\ell-1} i_{p}}^{1+i_{\ell}}\right) \varphi_{n-\sum_{p=1}^{w} i_{p}}^{k-\sum_{p}^{w} i_{p}} \\
& \quad=\hat{h}_{u+w}\left(\sigma^{n}(a), \sigma^{n-k}(a)\right) .
\end{aligned}
$$

For an easier work, we introduce the multiindex notation, namely,

$$
\begin{aligned}
& i=\left(i_{1}, i_{2}, \ldots, i_{w}\right), \quad|i|_{\ell}=\sum_{p=1}^{\ell} i_{p}, \quad \ell \in\{1,2, \ldots, w\}, \\
& |i|=|i|_{w} \quad \text { and } \quad \bar{i}=\max _{\ell \in\{1, \ldots, w\}} i_{\ell} .
\end{aligned}
$$

Then we can write shortly

$$
\sum_{0 \leq|i| \leq k-1} \varphi_{n-|i|}^{k-|i|} \prod_{\ell=1}^{w} v\left(\sigma^{n-|i|_{\ell}}(a)\right) \varphi_{n-|i|_{\ell-1}}^{i_{\ell}+1}=\hat{h}_{u+w}\left(\sigma^{n}(a), \sigma^{n-k}(a)\right) .
$$

Now, we extract the summands involving $\varphi_{n}^{k}$, which are characterized by $|i|=0$ or $\bar{i}=k-1$, i.e.,

$$
\begin{aligned}
& \varphi_{n}^{k}\left(v^{w}\left(\sigma^{n}(a)\right)\left(\varphi_{n}^{1}\right)^{w}+\varphi_{n-k+1}^{1} \sum_{\ell=1}^{w} v^{w-\ell+1}\left(\sigma^{n-k+1}(a)\right) v^{\ell-1}\left(\sigma^{n}(a)\right)\left(\varphi_{n-k+1}^{1}\right)^{w-\ell}\left(\varphi_{n}^{1}\right)^{\ell-1}\right) \\
& +\sum_{0<|i| \leq k-1}^{\bar{i} \neq k-1} \varphi_{n-|i|}^{k-|i|} \prod_{\ell=1}^{w} v\left(\sigma^{n-|i|_{\ell}}(a)\right) \varphi_{n-|i|_{\ell-1}}^{i_{\ell}}=\hat{h}_{u+w}\left(\sigma^{n}(a), \sigma^{n-k}(a)\right) .
\end{aligned}
$$

Evaluating the terms $\varphi^{1}$. by (15), we can express $\hat{h}_{r}\left(t, \rho^{k}(t)\right)=\varphi_{n}^{k}\left(t=\sigma^{n}(a)\right)$ and arrive at recursion

$$
\begin{aligned}
& \hat{h}_{r}\left(t, \rho^{k}(t)\right) \\
& \quad=\frac{\hat{h}_{u+w}\left(t, \rho^{k}(t)\right)-\sum_{0<i|| \leq k-1}^{i \neq k-1} \hat{h}_{r}\left(\rho^{|i|}(t), \rho^{k}(t)\right) \prod_{\ell=1}^{w} v\left(\rho^{|i| \ell}(t)\right) \hat{h}_{r}\left(\rho^{|i| l_{\ell-1}}(t), \rho^{|i| l_{\ell}+1}(t)\right)}{\sum_{\ell=0}^{w} v^{(r+1) \ell}(t) v^{(r+1)(w-\ell)}\left(\rho^{k-1}(t)\right)}, \\
& \quad k>1,
\end{aligned}
$$

which, supplied with (15), determines uniquely $\hat{h}_{r}(t, s)$ for all $r \in(-1, \infty)_{\mathbb{Q}}, s, t \in \mathbb{T}$ such that $t>s$. 
Remark 4.4 As a byproduct of the proof of Theorem 4.3, we have obtained the recursive formula (16). Hence, employing (15), (16), there can be created an effective algorithm evaluating numerically the power functions of rational orders on a given isolated time scale. Note that for special isolated time scales such that $v(t) \neq v(s)$ for all $s, t \in \mathbb{T}_{\kappa}$, Lemma 4.1 yields a more simple formula

$$
\hat{h}_{r}(t, s)=\frac{v(t) \hat{h}_{r}(t, \sigma(s))-v(\sigma(s)) \hat{h}_{r}(\rho(t), s)}{\nu(t)-v(\sigma(s))} .
$$

Remark 4.5 Definition 3.1 prescribes nonnegative values of $\hat{h}_{\beta}(t, s)$. Although so far known power functions fulfilling (15), (16) have this property (i.e., power functions on $\mathbb{R}, h \mathbb{Z}, \overline{q^{\mathbb{Z}}}$ and $\mathbb{T}_{(q, h)}$, as well as the ones obtained numerically), a general proof of nonnegativity of solutions of (15), (16) is still an open problem. However, since (15), (16) represent the unique functions satisfying Definition 3.1, an occurrence of a negative value of a power function on some time scale would imply a reevaluation of the nonnegativity condition.

Theorem 4.6 Let $\mathbb{T}$ be an isolated time scale, let $t \in \mathbb{T}$, and let $r \in(-1, \infty)_{\mathbb{Q}}$. Then

(i) $\hat{h}_{r}(t, t)=0$ for $r>0$,

(ii) $\hat{h}_{r}(t, t)=1$ for $r=0$,

(iii) the value of $\hat{h}_{r}(t, t)$ is unbounded for $-1<r<0$.

Proof First, let $r=0$. Then $\hat{h}_{0}(t, t)=1$, as prescribed by Definition 3.1(ii). Let $r>0$. For $0<r<1$, the zero value is given by Definition 3.1(iii). For $r \geq 1$, the assertion follows by subsequent application of the relation $\hat{h}_{r}(t, t)=\int_{t}^{t} \hat{h}_{r-1}(\tau, t) \nabla \tau=0$, where the zero value is implied by (4). Finally, let $-1<r<0$. Since $-r-1>-1$, we can write by Definition 3.1(i), (ii) $\int_{t}^{t} \hat{h}_{-r-1}(t, \rho(\tau)) \hat{h}_{r}(\tau, t) \nabla \tau=\hat{h}_{0}(t, t)=1$. Considering (4) and (15), we conclude that the value of $\hat{h}_{r}(t, t)$ is unbounded.

Remark 4.7 (i) Theorem 4.6 corresponds with the continuous case, where power functions $\frac{(t-s)^{r}}{\Gamma(r+1)}, r \in(-1,0)$ are unbounded in a neighborhood of $t=s$. It is known that in the continuous case, we can utilize an improper integration to calculate $\int_{t}^{t} \frac{(\tau-t)^{r}}{\Gamma(r+1)} \mathrm{d} \tau=0$. On isolated time scales, it follows by Definition 3.1(iii).

(ii) The power functions of order $r \in(-1,0)_{\mathbb{Q}}$ represent a new class of 'singular' functions on time scales. Besides continuous calculus, this phenomenon appeared, e.g., in [9], where the singular functions were introduced on $\overline{q^{\mathbb{Z}}}$ with a singularity at the cluster point.

(iii) Considering Remark 3.4, power functions of orders $r \in(-2,-1)$ have unbounded values $\hat{h}_{r}(t, t)$ and $\hat{h}_{r}(t, \rho(t))$. It implies that such functions are not integrable, which agrees with the continuous analysis.

Corollary 4.8 Let $\gamma>0, r \in(-1,0)_{\mathbb{Q}}, a \in \mathbb{T}$ and $\mathbb{T}$ be an isolated time scale. Then it holds

(i) $\left.{ }_{a} \nabla^{-\gamma} \hat{h}_{r}(t, a)\right|_{t=a}=0$ for $\gamma>-r$,

(ii) $\left.{ }_{a} \nabla^{-\gamma} \hat{h}_{r}(t, a)\right|_{t=a}=1$ for $\gamma=-r$,

(iii) $\left.{ }_{a} \nabla^{-\gamma} \hat{h}_{r}(t, a)\right|_{t=a}$ is unbounded for $\gamma<-r$.

Proof The assertion follows by Theorems 3.8 and 4.6.

To illustrate and clarify the notion of the power functions on time scales, we conclude this section by the following examples. 
Example 4.9 It can be proved that Definition 3.1 (and also recursive formulas (15), (16)) is satisfied by the power functions $\hat{h}_{r}(t, s)$, historically established on $\mathbb{R}, h \mathbb{Z}, \overline{q^{\mathbb{Z}}}$ and $\mathbb{T}_{(q, h)}$ provided $t>s$ (this matter was discussed, e.g., in [6]). We demonstrate this on the case $\mathbb{T}=\mathbb{Z}$, when Definition 3.1 yields

$$
\hat{h}_{r}(t, s)=\left\{\begin{array}{ll}
\left(\begin{array}{c}
k+r-1 \\
k-1
\end{array}\right), & k>0, \\
0, & k=0, \\
\text { undefined, } & k<0,
\end{array} \quad \text { where } r \in(0, \infty)_{\mathbb{Q}}, t=\sigma^{k}(s) .\right.
$$

Indeed, properties $(17)_{2}$ and $(17)_{3}$ are directly implied by Theorem 4.6(i) and Definition 3.1, respectively. For $r \in \mathbb{Z}^{+}$, the property (17) is reduced into the classical result known from the theory of time scales monomials. To confirm the validity of $(17)_{1}$ also for rational values of $r$, we employ the induction principle. Applying (15), we can easily verify the case $k=1$. Now, denote $r=\frac{u}{w+1}\left(u, w \in \mathbb{Z}^{+}\right)$and assume that (17) $)_{1}$ holds for $k-1$. Thus, (16) yields

$$
\begin{aligned}
& \hat{h}_{\frac{u}{w+1}}(n, n-k) \\
& \quad=\frac{1}{w+1}\left(\left(\begin{array}{c}
k+u+w-1 \\
k-1
\end{array}\right)-\sum_{0<\langle i| \leq k-1}^{i \neq k-1}\left(\begin{array}{c}
k-|i|+\frac{u}{w+1}-1 \\
k-|i|-1
\end{array}\right) \prod_{\ell=1}^{w}\left(\begin{array}{c}
|i|_{\ell}-|i|_{\ell-1}+\frac{u}{w+1} \\
|i|_{\ell}-|i|_{\ell-1}
\end{array}\right)\right) .
\end{aligned}
$$

Treating the occurring summation $\sum_{0<|i| \leq k-1}^{\bar{i} \neq k-1}$ is in general technically quite difficult. Hence, for the sake of simplicity, we perform the calculations only for $w=2$. We get

$$
\begin{aligned}
\hat{h}_{\frac{u}{3}}(n, n-k)= & \frac{1}{3}\left(\left(\begin{array}{c}
k+u-1 \\
k-1
\end{array}\right)-\sum_{i_{1}=1}^{k-2}\left(\begin{array}{c}
i_{1}+\frac{u}{3} \\
i_{1}
\end{array}\right) \sum_{i_{2}=0}^{k-1-i_{1}}\left(\begin{array}{c}
k-i_{1}-i_{2}-1+\frac{u}{3} \\
k-i_{1}-i_{2}-1
\end{array}\right)\left(\begin{array}{c}
i_{2}+\frac{u}{3} \\
i_{2}
\end{array}\right)\right. \\
& \left.-\sum_{i_{2}=1}^{k-2}\left(\begin{array}{c}
k-i_{2}-1+\frac{u}{3} \\
k-i_{2}-1
\end{array}\right)\left(\begin{array}{c}
i_{2}+\frac{u}{3} \\
i_{2}
\end{array}\right)\right) \\
= & \frac{1}{3}\left(\left(\begin{array}{c}
k+u-1 \\
k-1
\end{array}\right)-\sum_{i_{1}=1}^{k-2}\left(\begin{array}{c}
i_{1}+\frac{u}{3} \\
i_{1}
\end{array}\right)\left(\begin{array}{c}
k-i_{1}-1+\frac{u}{3} \\
k-i_{1}-1
\end{array}\right)\right. \\
& \left.-\left(\begin{array}{c}
k-1+2 \frac{u}{3} \\
k-1
\end{array}\right)+2\left(\begin{array}{c}
k-1+\frac{u}{3} \\
k-1
\end{array}\right)\right) \\
= & \frac{1}{3}\left(\begin{array}{c}
k+u-1 \\
k-1
\end{array}\right)-\left(\begin{array}{c}
k-1+3 \frac{u}{3} \\
k-1
\end{array}\right)+\left(\begin{array}{c}
k-1+2 \frac{u}{3} \\
k-1
\end{array}\right) \\
& \left.-\left(\begin{array}{c}
k-1+2 \frac{u}{3} \\
k-1
\end{array}\right)+3\left(\begin{array}{c}
k-1+\frac{u}{3} \\
k-1
\end{array}\right)\right) \\
= & \left(\begin{array}{c}
k+\frac{u}{3}-1 \\
k-1
\end{array}\right),
\end{aligned}
$$

where we utilize the well-known Vandermonde's convolution [23, formula (6:5:5)] twice. Note that similar technique can be utilized also for power functions on $h \mathbb{Z}, \overline{q^{\mathbb{Z}}}$ and $\mathbb{T}_{(q, h)}$ provided the corresponding version of Vandermonde's convolution is employed. 
Example 4.10 We consider isolated time scales

$$
\begin{aligned}
& \mathbb{T}_{1}=\mathbb{Z}=\left\{t_{n} ; t_{n}=n, n \in \mathbb{Z}\right\}, \\
& \mathbb{T}_{2}=\left\{t_{n} ; t_{n}=0.2 \cdot\left\lceil\frac{n}{2}\right\rceil+\left\lfloor\frac{n}{2}\right\rfloor, n \in \mathbb{Z}\right\}, \quad \text { where }\lfloor\xi\rfloor=\max \{m \in \mathbb{Z} ; m \leq \xi\}, \\
& \mathbb{T}_{3}=\left\{t_{n} ; t_{n}=n \text { for } n \leq 4 \text { and } t_{n}=4+0.2 \cdot(n-4) \text { for } n \geq 5, n \in \mathbb{Z}\right\}, \\
& \mathbb{T}_{4}=\left\{t_{n} ; t_{n} \text { are randomly generated points such that } 0 \leq t_{n} \leq 5, n \in \mathbb{Z}\right\}, \\
& \mathbb{T}_{5}=\left\{t_{n} ; t_{n}=0.1 \cdot n \text { for } n \leq 10 \text { and } t_{n}=2.5+0.3 \cdot(n-11) \text { for } n \geq 11, n \in \mathbb{Z}\right\} .
\end{aligned}
$$

For these time scales, we depict the power functions of orders $-\frac{1}{2}$ and $\frac{1}{3}$. For a better comparison, we plot at Figures 1-4 also their differences with respect to power function in $\mathbb{R}$. We can observe a trend of tending to the values of continuous power functions with decreasing values of graininess and increasing $t$.
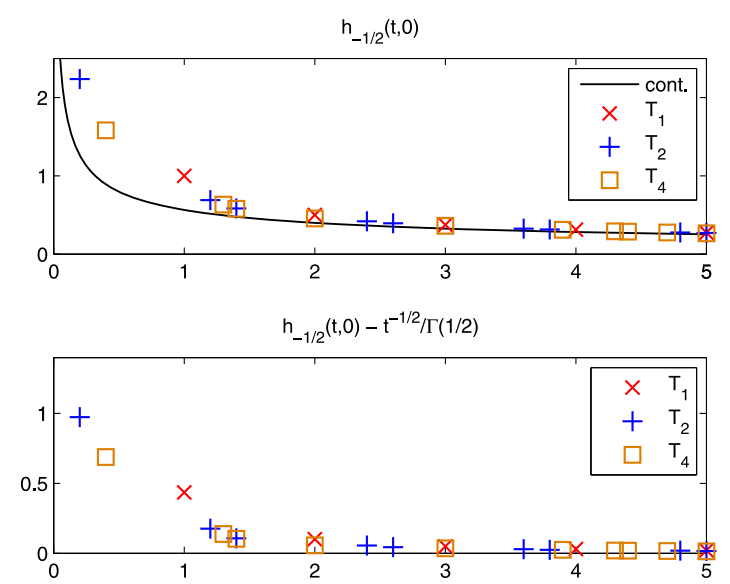

Figure $1 \hat{h}_{-\frac{1}{2}}(t, 0)$ on $\mathbb{T}_{1}, \mathbb{T}_{2}$ and $\mathbb{T}_{4}$.
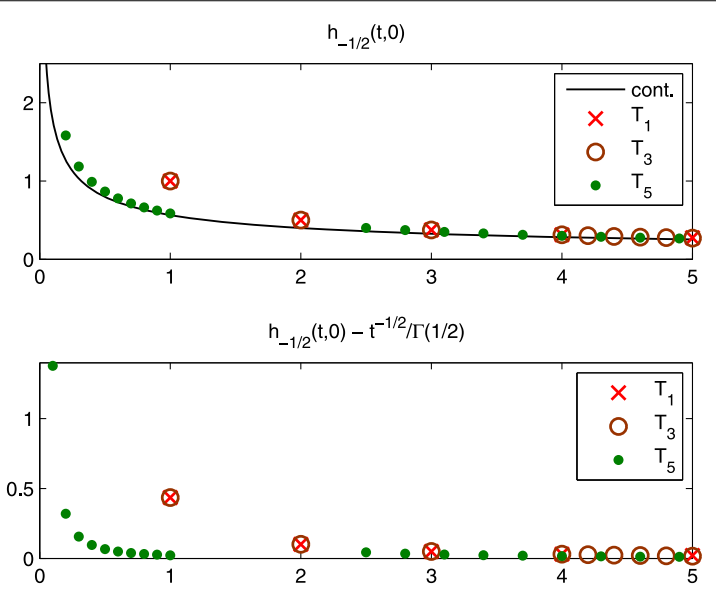

Figure $2 \hat{h}_{-\frac{1}{2}}(t, 0)$ on $\mathbb{T}_{1}, \mathbb{T}_{3}$ and $\mathbb{T}_{5}$. 

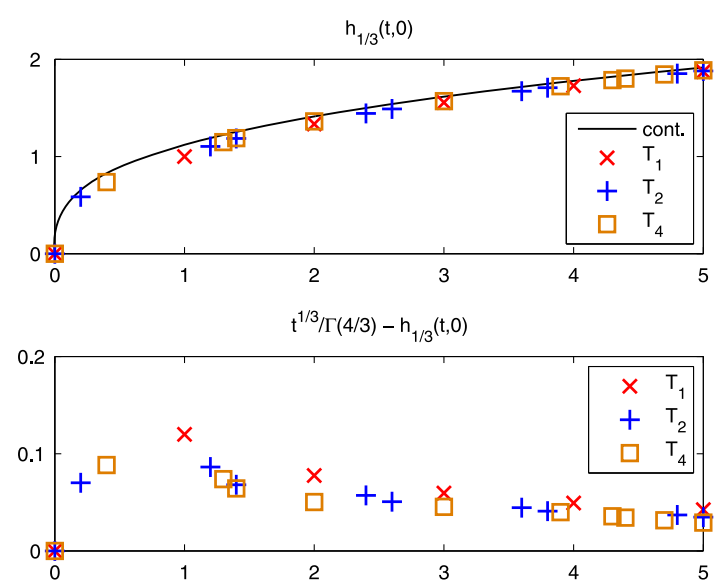

Figure $3 \hat{h}_{\frac{1}{3}}(t, 0)$ on $\mathbb{T}_{1}, \mathbb{T}_{2}$ and $\mathbb{T}_{4}$.
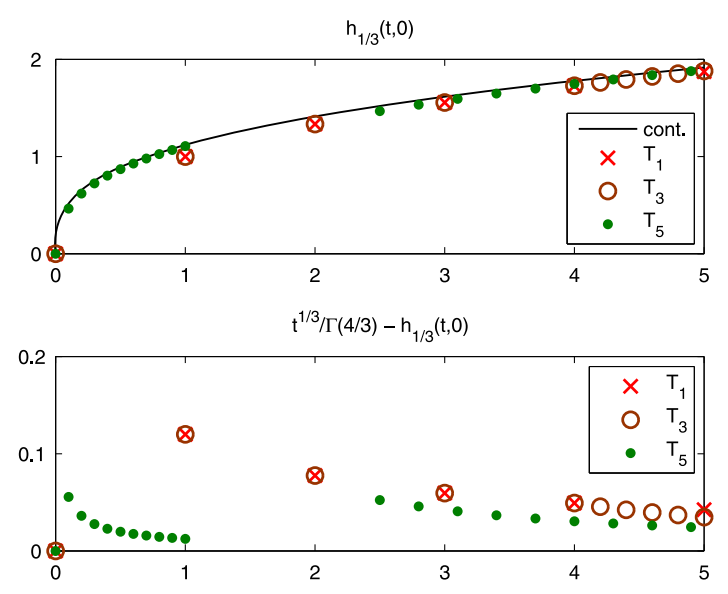

Figure $4 \hat{h}_{\frac{1}{3}}(t, 0)$ on $\mathbb{T}_{1}, \mathbb{T}_{3}$ and $\mathbb{T}_{5}$.

\section{Laplace transform}

The Laplace transform is a tool of a key importance regarding solving and analyzing of fractional differential equations in the continuous (see, e.g., $[1,2]$ ), as well as in the discrete case (see, e.g., [7] and [14]). In this section, we deal with the Laplace transform of the power functions on isolated time scales $\mathbb{T}_{\mathcal{L}}$ (see Definition 2.1), which implies also the Laplace transforms of fractional operators. We demonstrate a usage of the Laplace transform method for solving of initial value problems by a simple example.

Theorem 5.1 Let $a \in \mathbb{T}_{\mathcal{L}}$, and let $r \in(-1, \infty)_{\mathbb{Q}}$. Then it holds

$$
\mathcal{L}_{a}\left\{\hat{h}_{r}(\cdot, a)\right\}(z)=z^{-r-1} .
$$

Proof Let $r=\frac{u}{w+1}\left(u \in \mathbb{Z}, w \in \mathbb{Z}^{+},-u \leq w\right)$. It is known that $\mathcal{L}_{a}\left\{\hat{h}_{u+w}(\cdot, a)\right\}(z)=z^{-u-w-1}$ (see (11)). Assume that $g$ is a function such that $\mathcal{L}_{a}\{g\}(z)=z^{-r-1}$. Then Theorem $2.6 \mathrm{im}$ - 
plies

$$
\begin{gathered}
\mathcal{L}_{a}\{(\underbrace{g * \cdots * g}_{(w+1) \times})(\cdot, a)\}(z)=\left(z^{-r-1}\right)^{w+1}=\mathcal{L}_{a}\left\{\hat{h}_{u+w}\right\}(z) \\
\Longrightarrow \quad \underbrace{(g * \cdots * g}_{(w+1) \times})(t, a)=\hat{h}_{u+w}(t, a) .
\end{gathered}
$$

On the other hand, a subsequent application of Definition 3.1(i) (see Remark 4.2) yields

$$
(\underbrace{\hat{h}_{r} * \cdots * \hat{h}_{r}}_{(w+1) \times})(t, a)=\hat{h}_{u+w}(t, a) .
$$

Theorem 4.3 guarantees the uniqueness of $\hat{h}_{r}$, therefore, it follows

$$
\hat{h}_{r}(t, a)=g(t) \quad \Longrightarrow \quad \mathcal{L}_{a}\left\{\hat{h}_{r}(\cdot, a)\right\}(z)=z^{-r-1},
$$

which concludes the proof.

Now, we can write the time scales analogues of some relations of continuous fractional calculus.

Corollary 5.2 Let $\gamma, \alpha \in(0, \infty)_{\mathbb{Q}}, a \in \mathbb{T}_{\mathcal{L}}$ and $f: \mathbb{T}_{\mathcal{L}} \rightarrow \mathbb{R}$ be such that $\mathcal{L}_{a}\{f\}(z)$ exists. Then it holds

(i) $\mathcal{L}_{a}\left\{{ }_{a} \nabla^{-\gamma} f\right\}(z)=z^{-\gamma} \mathcal{L}_{a}\{f\}(z)$,

(ii) $\mathcal{L}_{a}\left\{\nabla^{\alpha} f\right\}(z)=z^{\alpha} \mathcal{L}_{a}\{f\}(z)-\left.\sum_{j=0}^{\lceil\alpha\rceil-1} z_{a}^{j} \nabla^{\alpha-j-1} f(t)\right|_{t=a}$,

(iii) $\mathcal{L}_{a}\left\{{ }_{a}^{C} \nabla^{\alpha} f\right\}(z)=z^{\alpha} \mathcal{L}_{a}\{f\}(z)-\left.\sum_{j=0}^{\lceil\alpha\rceil-1} z^{\alpha-\lceil\alpha\rceil+j}{ }_{a}^{\lceil\alpha\rceil-j-1} f(t)\right|_{t=a}$.

Proof All three formulas are direct consequences of Theorem 5.1, supplied with (11), (12), Theorems 2.6 and 5.1. The proofs are analogous to the continuous paradigm (see, e.g., $[2])$.

Remark 5.3 In [16], the authors introduce the fractional operators via the Laplace transform in the frame of the delta calculus. Note that this idea is transferable also to the nabla case. In this sense, Corollary 5.2 shows that Definition 3.1 is consistent for special time scales $\mathbb{T}_{\mathcal{L}}$ with the approach utilized in [16].

Corollary 5.2 enables us to utilize the Laplace transform method for an effective solving of some initial value problems. We point out that such results are not only symbolical, but due to (15), (16), they have a solid meaning, and it is possible to plot the solutions on every isolated time scale. We demonstrate this method by the following example.

Example 5.4 Let $\alpha \in(0,1]_{\mathbb{Q}}$, $a \in \mathbb{T}_{\mathcal{L}}$. Consider the pair of initial value problems with Riemann-Liouville and Caputo fractional operators

$$
\begin{array}{ll}
{ }_{a} \nabla^{\alpha} y(t)=\frac{4}{5}, \quad t>a, & { }_{a}^{C} \nabla^{\alpha} y(t)=\frac{4}{5}, \quad t>a, \\
\left.{ }_{a} \nabla^{\alpha-1} y(t)\right|_{t=a}=y_{\alpha-1}, & y(a)=y_{0},
\end{array}
$$


where $y_{\alpha-1}, y_{0} \in \mathbb{R}$. Note that the initial condition $\left.{ }_{a} \nabla^{\alpha-1} y(t)\right|_{t=a}=y_{\alpha-1}$ can be interpreted via Corollary 4.8 (see also [14]). Utilizing the Laplace transform, namely Corollary 5.2 and (11), we arrive at

$$
\mathcal{L}_{a}\left\{y_{\mathrm{RL}}\right\}(z)=\frac{4}{5} \frac{1}{z^{1+\alpha}}+y_{\alpha-1} \frac{1}{z^{\alpha}}, \quad \mathcal{L}_{a}\left\{y_{\mathrm{C}}\right\}(z)=\frac{4}{5} \frac{1}{z^{1+\alpha}}+y_{0} \frac{1}{z} .
$$

Applying Theorem 5.1, we obtain the solutions

$$
y_{\mathrm{RL}}(t)=\frac{4}{5} \hat{h}_{\alpha}(t, a)+y_{\alpha-1} \hat{h}_{\alpha-1}(t, a), \quad y_{\mathrm{C}}(t)=\frac{4}{5} \hat{h}_{\alpha}(t, a)+y_{0} \hat{h}_{0}(t, a) .
$$

We point out that we would get the same results also for $\mathbb{T}=\mathbb{R}$. In Figures 5 and 6 , we plot the solutions on time scales $\mathbb{T}_{1}, \mathbb{T}_{2}$ and $\mathbb{T}_{5}$ introduced in Example 4.10.

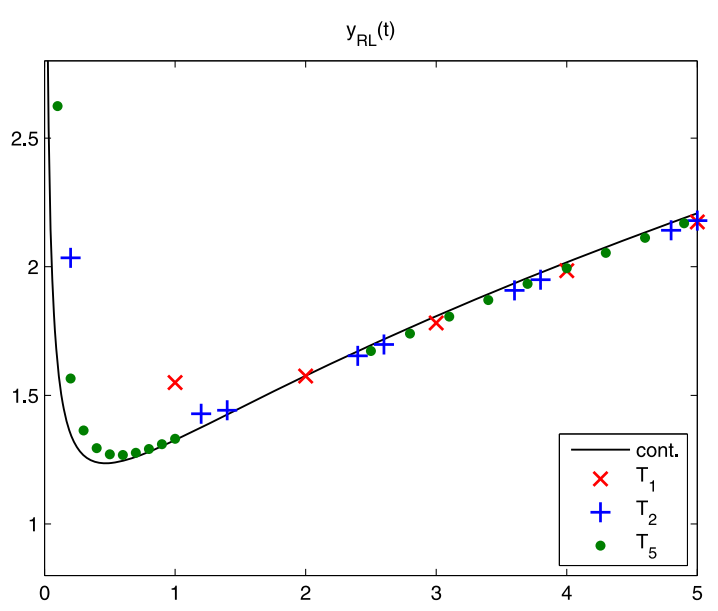

Figure $5 y_{\mathrm{RL}}(t)$ for $\alpha=\frac{1}{3}, y_{-\frac{2}{3}}=\frac{3}{4}$.

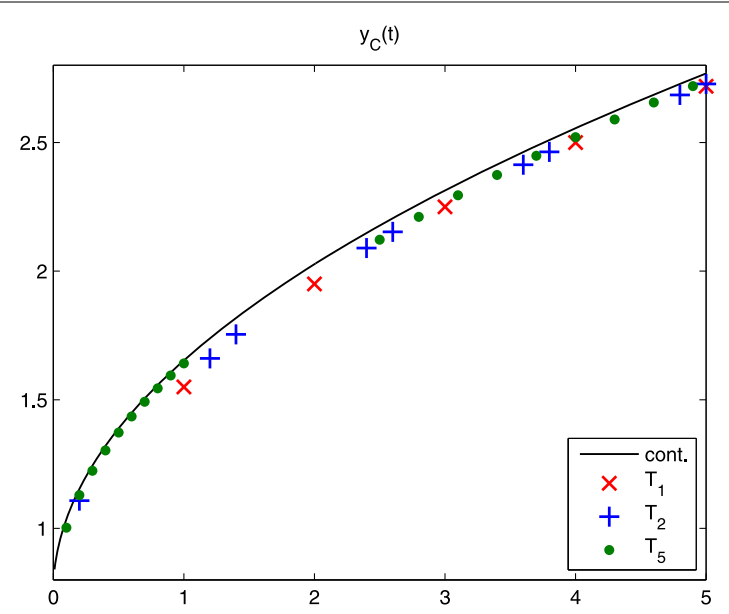

Figure $6 y_{\mathrm{C}}(t)$ for $\alpha=\frac{1}{3}, y_{0}=\frac{3}{4}$. 


\section{Conclusions and open problems}

Fractional calculus on time scales is a new topic providing many directions for further research. In this paper, we have discussed relations of the axiomatic approach, represented by Definitions 3.1 and 3.5 to the work of the other authors, namely, $[6,16]$. The key assertion, i.e., Theorem 4.3 concerning existence and uniqueness of power functions, was derived under the assumption of rational orders and isolated time scales. This result has many important consequences, e.g., Theorem 5.1, therefore, its extension for time scales, involving dense points or for irrational orders of power functions, seems to be very desirable.

Regarding the values of the power functions, we have introduced the recursive formulas (15), (16). Besides the question of the nonnegativity (see Remark 4.5), it remains an open problem if a corresponding relation exists also in a closed form. Considering the power functions of negative orders, we have also mentioned a notion of singular time scales functions, a matter which is quite unexplored so far (see Remark 4.7).

A solving of more advanced fractional differential equations on time scales requires an introduction of analogues of various special functions utilized in the continuous fractional calculus, e.g., a dynamic Mittag-Leffler function $E_{\eta, \beta}^{\lambda}(t, s)=\sum_{j=0}^{\infty} \lambda^{j} \hat{h}_{\eta j+\beta-1}(t, s)$ (see, e.g., [2, $10]$ and [12]). A question of convergence of the related infinite series, as well as many other topics such as stability analysis, is closely connected to an issue of asymptotic properties of the general dynamic power functions. We conjecture that the estimate

$$
v_{\max }^{r}(t)\left(\begin{array}{c}
n+r-1 \\
n-1
\end{array}\right) \leq \hat{h}_{r}(t, s) \leq v_{\min }^{r}(t)\left(\begin{array}{c}
n+r-1 \\
n-1
\end{array}\right), \quad r \in(-1,0)_{\mathbb{Q}}, t=\sigma^{n}(s)
$$

holds, where $v_{\max }(t)=\max \left\{v(\tau) ; \tau \in(s, t]_{\mathbb{T}}\right\}$ and $v_{\min }(t)=\min \left\{v(\tau) ; \tau \in(s, t]_{\mathbb{T}}\right\}$. However, its general proof is not available so far, therefore, it remains in a form of hypothesis.

Competing interests

The author declares that he has no competing interests.

\section{Author's contributions}

TK is the only author of this paper. The author read and approved the final manuscript.

\section{Acknowledgements}

The research was supported by the project CZ.1.07/2.3.00/30.0039 of Brno University of Technology.

Received: 1 March 2013 Accepted: 29 July 2013 Published: 23 August 2013

References

1. Kilbas, AA, Srivastava, HM, Trujillo, JJ: Theory and Applications of Fractional Differential Equations. Elsevier, Amsterdam (2006)

2. Podlubny, I: Fractional Differential Equations. Academic Press, New York (1999)

3. Agarwal, RP: Certain fractional q-integrals and q-derivatives. Math. Proc. Camb. Philos. Soc. 66, 365-370 (1969)

4. Diaz, R, Osler, TJ: Differences of fractional order. Math. Comput. 28, 185-202 (1974)

5. Čermák, J, Nechvátal, L: On (q,h)-analogue of fractional calculus. J. Nonlinear Math. Phys. 17, 51-68 (2010)

6. Williams, PA: Fractional calculus on time scales with Taylor's theorem. Fract. Calc. Appl. Anal. 15, 616-638 (2012)

7. Atici, FM, Eloe, PW: A transform method in discrete fractional calculus. Int. J. Differ. Equ. 2, 165-176 (2007)

8. Atici, FM, Eloe, PW: Discrete fractional calculus with the nabla operator. Electron. J. Qual. Theory Differ. Equ. 2009, 3 (2009)

9. Mansour, ZSI: Linear sequential q-difference equations of fractional order. Fract. Calc. Appl. Anal. 12, 159-178 (2009)

10. Nagai, A: On a certain fractional q-difference and its eigen function. J. Nonlinear Math. Phys. 10, 133-142 (2003)

11. Rahmat, MRS: On some $(q, h)$-analogues of integral inequalities on discrete time scales. Comput. Math. Appl. 62 1790-1797 (2011)

12. Čermák, J, Kisela, T, Nechvátal, L: Discrete Mittag-Leffler functions in linear fractional difference equations. Abstr. Appl. Anal. 2011, Article ID 565067 (2011)

13. Čermák, J, Kisela, T, Nechvátal, L: Stability and asymptotic properties of a linear fractional difference equation. Adv. Differ. Equ. 2012, 122 (2012) 
14. Čermák, J, Kisela, T, Nechvátal, L: Stability regions for linear fractional differential systems and their discretizations. Appl. Math. Comput. 219, 7012-7022 (2013)

15. Anastassiou, GA: Foundations of nabla fractional calculus on time scales and inequalities. Comput. Math. Appl. 59 3750-3762 (2010)

16. Bastos, NRO, Mozyrska, D, Torres, DFM: Fractional derivatives and integrals on time scales via the inverse generalized Laplace transform. Int. J. Math. Comput. 11, 1-9 (2011)

17. Akin-Bohner, E, Bohner, M: Exponential functions and Laplace transforms for alpha derivatives. In: Proceedings of the Sixth International Conference on Difference Equations, Augsburg, Germany, pp. 231-238 (2001)

18. Kisela, T: Basics of qualitative theory of linear fractional difference equations. PhD thesis, Brno University of Technology, Faculty of Mechanical Engineering (2012)

19. Bohner, M, Peterson, A: Dynamic Equations on Time Scales: An Introduction with Applications. Birkhäuser, Boston (2001)

20. Bohner, M, Peterson, A: Advances in Dynamic Equations on Time Scales. Birkhäuser, Boston (2003)

21. Bohner, M, Peterson, A: Laplace transform and Z-transform: unification and extension. Methods Appl. Anal. 9, 155-162 (2002)

22. Bohner, M, Guseinov, GS: The convolution on time scales. Abstr. Appl. Anal. 2007, Article ID 58373 (2007)

23. Oldham, KB, Myland, J, Spanier, J: An Atlas of Functions. Springer, New York (2009)

doi:10.1186/1687-1847-2013-259

Cite this article as: Kisela: Power functions and essentials of fractional calculus on isolated time scales. Advances in Difference Equations 2013 2013:259.

\section{Submit your manuscript to a SpringerOpen ${ }^{\circ}$ journal and benefit from:}

- Convenient online submission

- Rigorous peer review

- Immediate publication on acceptance

Open access: articles freely available online

- High visibility within the field

- Retaining the copyright to your article 